\title{
The Fault Diagnosis of Smart Substation Equipment Based on Fuzzy Petri Nets
}

\author{
Shaowen Wan \\ College of Electrical Engineering and Automation, Qilu University of Technology (SDAS), Jinan \\ 250353, China \\ wonsoul@qq.com
}

Keywords: fuzzy Petri nets, fault diagnosis, hierarchical model, smart substation equipment.

\begin{abstract}
This paper introduces fault diagnosis method and its mathematical descriptionthe of fuzzy Petri nets, including the basic concept and rule representation. On the basis of the original fuzzy Petri net reasoning decision, the transition threshold judgment is proposed. This paper presents a fault diagnosis method for smart substation equipment based on fuzzy Petri nets, establishes a hierarchical fault diagnosis model, and simplifies the decision algorithm, which is validated by actual data.
\end{abstract}

\section{Introduction}

As the core of smart grid, smart substation is the most important component of smart grid, which shoulders the important task of power transmission and transformation. Because of the intelligence of primary equipment and the network of secondary systems, traditional monitoring and fault analysis methods cannot meet the needs of smart substation. It has become an important research direction to get a new fault analysis method to find out the fault cause or the fault component accurately and quickly after the equipment failure. Several commonly used fault diagnosis algorithms are expert system, artificial neural network, Bayesian network, optimization technology, support vector machine, Petri nets, etc. In addition, there are a variety of intelligent algorithms combined with diagnosis methods, which usually learn from each other for every diagnosis algorithms to achieve a better diagnosis effect.

Petri nets is a graphical reasoning tool for describing the generation and propagation of fault diagnosis. It can be combined with fuzzy reasoning, neural network, genetic algorithm and rough set. Many scholars adopt the method of combining fuzzy reasoning and Petri nets to solve the complex problems in the process of fault diagnosis, such as wireless sequence control system [2], compressor in petrochemical industry [3], power system [4-5] and so on. Some progress has been made. This paper introduces the reasoning decision of FPN (Fuzzy Petri Nets), and adds hierarchical decision. The modeling method is applied to the fault diagnosis of smart substation equipment, which simplifies the calculation and improves the accuracy and timeliness of fault detection.

\section{Mathematical Description of FPN}

\subsection{The Basic Concept of Fpn}

FPN is proposed on the basis of general Petri net theory and combined with fuzzy set theory. [6]In reference [3], the confidence threshold of transition rule is added to the reasoning decision of general fuzzy Petri nets. In reference [5], the hierarchical structure model is added to the general fuzzy Petri nets, but the confidence threshold of the transition rule is not set. Therefore, combining with the FPN rule in the general fuzzy Petri nets and the confidence threshold of the transition rule,this paper define FPN as a 7-tuple:

$$
S_{P F N}=\{P, R, \mathbf{I}, \mathbf{O}, \alpha, \beta, \Lambda\}
$$

In the formula (1),P $=\{\mathrm{P} 1, \mathrm{P} 2, \ldots, \mathrm{Pn}\}$, it means a set of finite libraries corresponds to fuzzy propositions, including the premises and conclusions of the rules. The algebra $\mathrm{n}$ means the total number of libraries. If $\mathrm{Pi}$ is a starting position, Pi means the starting repository. 
$\mathrm{R}=\{\mathrm{R} 1, \mathrm{R} 2, \ldots, \mathrm{Rm}\}$, it means a set of finite transitions corresponds to fuzzy rules. The algebra $\mathrm{m}$ means the total number of transitions.

I means a input matrix, $\mathrm{I}=(\mathrm{dij}) \mathrm{m} \times \mathrm{n}, \mathrm{dij}$ is a logical quantity, $\mathrm{dij} \in[0,1]$. When $\mathrm{Pi}$ is the input of $\mathrm{Rj}$ (There is a directed arc from Pi to $\mathrm{Rj}$.), the value of dij is the weight of the directed arc; When Pi is not the input of $\mathrm{Rj}, \mathrm{dij}=0 . \mathrm{i}=1,2, \ldots, \mathrm{m}, \mathrm{j}=1,2, \ldots, \mathrm{n}$.

$\mathrm{O}$ means an output matrix, $\mathrm{O}=(\mathrm{gij}) \mathrm{m} \times \mathrm{n}$, gij is a logical quantity,gij $\in[0,1]$. When $\mathrm{Pi}$ is the output of $\mathrm{Rj}$ (There is a directed arc from $\mathrm{Rj}$ to Pi.), the value of gij is the credibility of the rules; When Pi is not the output of $\mathrm{Rj}, \mathrm{gij}=0 . \mathrm{i}=1,2, \ldots, \mathrm{m}, \mathrm{j}=1,2, \ldots, \mathrm{n}$.

$\alpha: \mathrm{P} \rightarrow[0,1]$. It is a mapping of real numbers between 0 and 1 of the library, whose elements represent the confidence of the library and correspond to the logical state of fuzzy proposition.a 0 is the initial mark of Petri nets.

$\beta: \mathrm{R} \rightarrow[0,1]$.It is a mapping of real numbers between 0 and 1 of the transition, whose elements represent the confidence of the transition and correspond to the confidence of fuzzy rules. Taking Uas the confidence matrix of rules, $U=\operatorname{diag}(\beta 1, \beta 2, \ldots, \beta \mathrm{m})$.If $\beta \mathrm{j}=1$, the model is a general Petri net reasoning model without fuzzy variables.

$\Lambda: R \rightarrow[0,1]$.It is a mapping of real numbers between 0 and 1 of the transition, whose elements represent the threshold of the transition.If the token value of the transition input library (corresponding to the confidence degree of the proposition) is greater than the threshold, then the transition can be triggered, otherwise it cannot be triggered.

\subsection{Expression of Fuzzy Production Rules By Fpn}

General fault systems are complex and changeable. Because of the fuzziness, concurrency and spread of fault causes, the fuzzy corresponding relation between fault cause and fault event is usually expressed by fuzzy generation rule. After the threshold of transition is added, the traditional fuzzy relation rule generally has the following three basic forms:

Basic rule:if di then dj,when the corresponding transition of the rule is triggered, $\alpha(\mathrm{dj})=\alpha(\mathrm{di}) \times \beta \mathrm{i}$. This rule can be expressed in figure $1(\mathrm{a})$.

"and" rule:if dil $\wedge$ di $2 \wedge \ldots \wedge$ din then dj,when the corresponding transition of the rule is triggered,$\alpha(\mathrm{dj})=\min (\alpha(\mathrm{di1}), \alpha(\mathrm{di} 2), \ldots, \alpha(\operatorname{din})) \times \beta \mathrm{i}$. This rule can be expressed in figure $1(\mathrm{~b})$.

"or" rule:if di $\vee$ di $2 \vee \ldots \vee$ din then dj,when the corresponding transition of the rule is triggered,$\alpha(\mathrm{dj})=\max (\alpha(\mathrm{di} 1) \times \beta 1, \alpha(\mathrm{di} 2) \times \beta 2, \ldots, \alpha(\mathrm{din}) \times \beta \mathrm{in})$. This rule can be expressed in fig. $1(\mathrm{c})$.

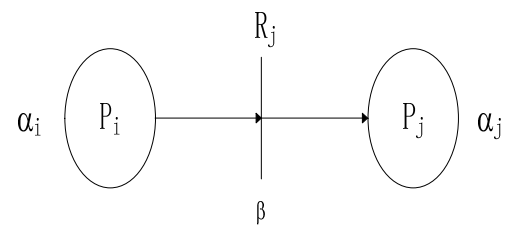

(a).basic rule

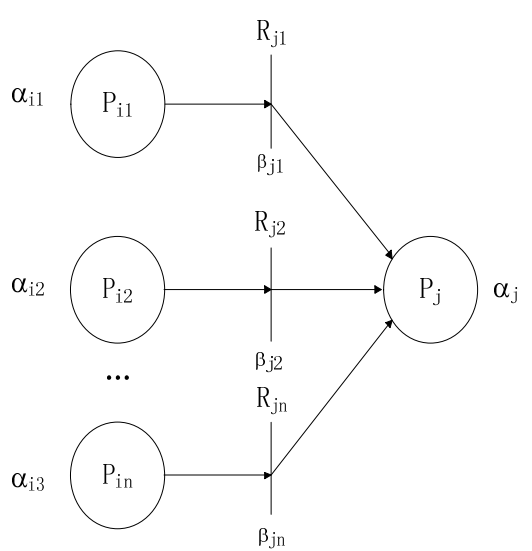

(b)."and" rule

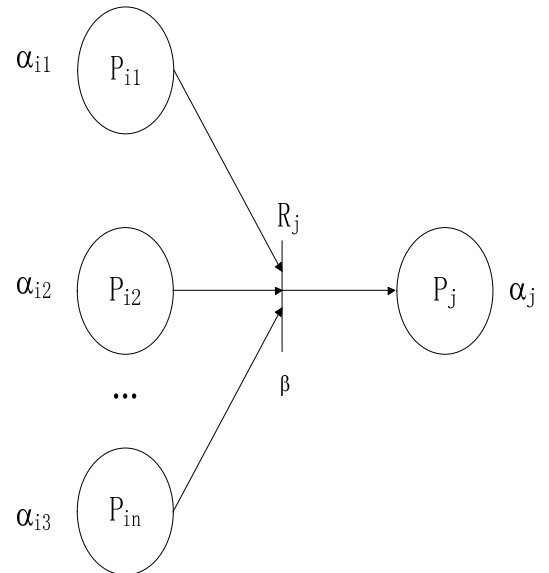

(c)."or" rule

Fig. 1 Representation of three fuzzy relations by Petri net 


\subsection{FPN Reasoning Decision With Transition Threshold Judgment}

The general FPN reasoning decision adopts the confidence degree method of MYCIN, which is a

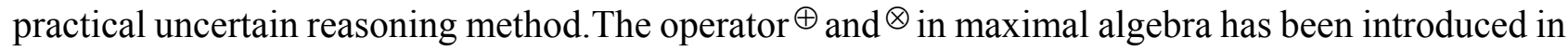
this method.

$$
\oplus: A \oplus B=C
$$

$\mathrm{A}, \mathrm{B}$ and $\mathrm{C}$ are all $\mathrm{m} \times \mathrm{n}$ matrices, $\mathrm{cij}=\min ($ aij,bij).

$$
\otimes: A \otimes B=D
$$

$\mathrm{A}$ is a $\mathrm{m} \times \mathrm{q}$ matric, $\mathrm{B}$ is a $\mathrm{q} \times \mathrm{n}$ matric, $\mathrm{C}$ is a $\mathrm{m} \times \mathrm{n}$ matric, $d_{i j}=\max _{1 \leq k \leq q}\left(a_{i k} b_{k j}\right)$.

Because of the addition of transition threshold in this paper,the operator $\odot$ and neg in reference [3] has been introduced.

$\mathrm{R}, \mathrm{S}$ and $\mathrm{Q}$ are all $\mathrm{m} \times \mathrm{n}$ matrices, qij $=\mathrm{rij} \cdot \mathrm{sij}$.

$$
\odot: R \odot S=Q
$$

$$
\text { neg : neg } \boldsymbol{\alpha}^{k}=\mathbf{1}_{m}-\boldsymbol{\alpha}^{k}=\overline{\boldsymbol{\alpha}^{k}}
$$

$1 \mathrm{~m}$ is a $\mathrm{m}$-dimensional vector,and all of its elements are 1 .The algebra $\mathrm{k}$ means reasoning steps.

According to the definitions of the above operators, the process of FPN reasoning decision with transition threshold judgment is as follows:

Step 1: Let $\mathrm{k}=0$.

Step 2:Figure out negak $=1 \mathrm{~m}-\mathrm{ak}$,negak is a $\mathrm{m}$-dimensional vector, whose elements represent the confidence of the proposition that the library $\mathrm{Pi}$ is false, $\mathrm{i}=1,2, \ldots, \mathrm{m}$.

Step 3:Calculate the intermediate variable $v \mathrm{k}, v \mathrm{k}=\mathrm{IT} \otimes($ negak), $v \mathrm{k}$ is a $\mathrm{n}$-dimensional vector,whose elements represent the confidence of the proposition that the rule $\mathrm{Rj}$ is false, $\mathrm{j}=1,2, \ldots, \mathrm{n}$.

Step 4:Figure out $\rho \mathrm{k}=\mathrm{neg} v \mathrm{k}=1 \mathrm{n}-\mathrm{vk}, \rho \mathrm{k}$ is a $\mathrm{n}$-dimensional vector, whose elements represent the confidence of the proposition that the rule $\mathrm{Rj}$ is true, $\mathrm{j}=1,2, \ldots, \mathrm{n}$.

Step 5:Judgment by comparing elements of $\rho \mathrm{k}$ and thresholds. $\mathrm{kk}=\rho \mathrm{k} \odot \operatorname{sign}(\rho \mathrm{k}-\Lambda), \varepsilon \mathrm{k}$ is a $n$-dimensional vector, whose elements represent the confidence of the proposition that the transition can be triggered is true.If $(\rho \mathrm{k}-\Lambda)>0$, sign $(\rho \mathrm{k}-\Lambda)=1$, otherwise it is 0 .

Step 6: The next state of library $\mathrm{Pi}$ is $\mathrm{ak}+1=\mathrm{ak} \oplus[(\mathrm{O} \cdot \mathrm{U}) \otimes \varepsilon \mathrm{k}]$.

Step 7: If $\mathrm{ak}+1 \neq \mathrm{ak}$, let $\mathrm{k}=\mathrm{k}+1$, repeat through step 2 to step 5 until $\mathrm{ak}+1=\mathrm{ak}$.

\section{Hierarchical Graphical Model For Fault Diagnosis By FPN}

Because of the intelligence of primary equipment in smart substation and the network of secondary system, the traditional methods of monitoring and fault analysis cannot meetthe demand of smart substation, which provides an opportunity for fault diagnosis by new method.FCK-851B/G Series Digital Measurement and Control device is suitable for smart substation of $750 \mathrm{kV}$ and below levels. The device is a new generation of measuring and controlling device that can support the application of smart substation. Taking the device as an example, the fault behavior database is established as follows.

P0: Line short or oxidation of node.

P1: Coil burned out.

P2: Node loosely or disconnected.

P3: Sampling pipe blockage.

P4: Electromagnetic valve broken.

P5: Belt slipped.

P6: Temperature sensor broken.

P7: Liquid level sensor broken.

P8: Actuator power lost.

P9: Motor seized up.

P10: Conveyor stop.

P11: The main board of control unit was damaged.

P12: Thread feedback error. 
P13: Measurements showed no data or fluctuations.

P14: Terminal controller alarmed.

According to the Petri net representation of three fuzzy relations in Section 1.1, the graphical modeling of the corresponding fault diagnosis is obtained, which is shown in the upper part of fig.2.

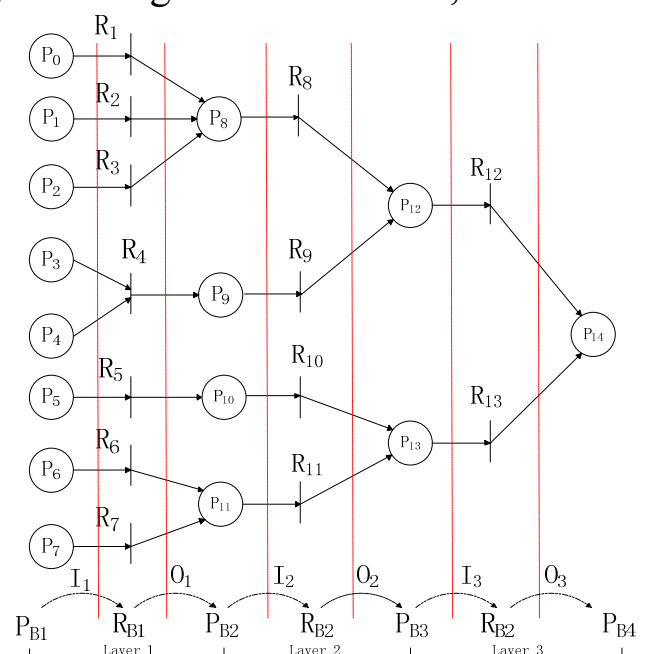

Fig.2 Hierarchical graphical model for FPN

According to the improvement of fuzzy Petri nets in reference [4],the model can be described in layers, as shown in the lower part of fig.2. Group the libraries and transitions in the model, $\mathrm{P}=\{\mathrm{PB} 1$, $\mathrm{PB} 2, \mathrm{~PB} 3, \mathrm{~PB} 4\}, \mathrm{R}=\{\mathrm{RB} 1, \mathrm{RB} 2, \mathrm{RB} 3\}$, where $\mathrm{PB} 1=\{\mathrm{P} 0, \mathrm{P} 1, \ldots, \mathrm{P} 7\}, \mathrm{PB} 2=\{\mathrm{P} 8, \mathrm{P} 9, \mathrm{P} 10, \mathrm{P} 11\}, \mathrm{PB} 3=\{$

$\mathrm{P} 12, \mathrm{P} 13\}, \mathrm{PB} 4=\{\mathrm{P} 14\}, \mathrm{RB} 1=\{\mathrm{R} 1, \mathrm{R} 2, \ldots, \mathrm{R} 7\}, \mathrm{RB} 2=\{\mathrm{R} 8, \mathrm{R} 9, \mathrm{R} 10, \mathrm{R} 11\}, \mathrm{RB} 3=\{\mathrm{R} 12, \mathrm{R} 13\}$. The

advantage of hierarchical graphical model is that it can express the direction of fault spread intuitively and clearly, and it has good adaptability in expanding. If there is new fault information, it can be assigned to the corresponding layer according to the location, thus saving the time of redrawing.

The input and output matrices, whose sub-correlation hierarchical matrices is separately framed in a rectangular frame, associated with the model are shown in fig.3.It can be seen that the sub-correlation hierarchical matrices are non-zero matrices in the associated matrix, ignoring a lot of no-practical elements, which effectively reduce the dimension of the reasoning matrix and reduce the computation cost.
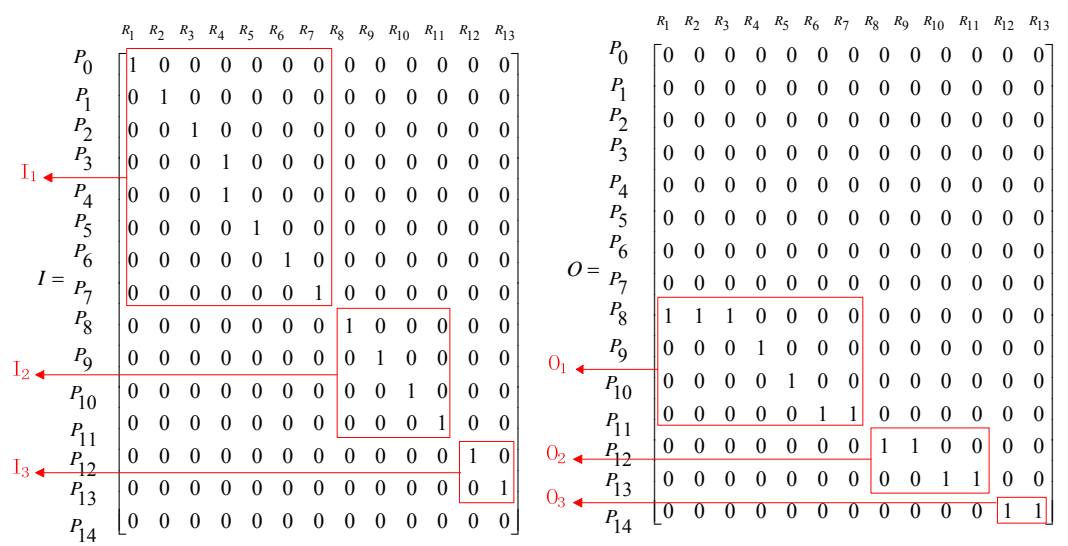

Fig. 3 Input and Output correlation matrix of fault diagnosis model

\section{Model Reasoning Decision Improvement and Data Calculation}

The hierarchical transition is carried out in order, that is, the second layer is triggered after the first layer transition is triggered, until finally. Combined with the reasoning decision of FPN in section 1.3, the state transition of each layer library needs to be calculated once. After three operations, the desired results are calculated. 
In order to reduce the amount of calculation, the FPN reasoning decision with transition threshold judgment is simplified, and the actual data in the process of using FCK-851-B digital microcomputer measurement and control device are placed in each matrix.

The vector a 0 with 15 -dimensions is improved to a vector group consisting of the initial state corresponding to the four-layer libraries. That is $\mathrm{a} 0=(\mathrm{a} 01, \mathrm{a} 02, \mathrm{a} 03, \mathrm{a} 04)$, where $\mathrm{a} 01=(0.75,0.3,0.6$, $0.7,0$,

$0.8,0.6,0.6) . a 01$ is an 8-dimensional vector, consisting of the initial state of the PB1 group in the libraries. And the other, a02/a03/a04 is a 4-dimensional/2-dimensional/1-dimensional vector, consisting of the initial state of thePB2/PB3/PB4 group in the libraries, all of them are all zero element vectors.

The threshold vector $\Lambda$ with 13-dimensions is improved to a vector group consisting of the thresholds of the three-layer transitions. That is $\Lambda=(\Lambda 1, \Lambda 2, \Lambda 3)$, where $\Lambda 1=(0.4,0.5,0.5,0.6,0.4,0.3,0.3)$,

$\Lambda 2=(0.5,0.3,0.4,0.3), \Lambda 3=(0.2,0.1)$.

The vector $U$ with 13-dimensions is improved to a vector group consisting of the confidence of the three-layer transitions. That is $\mathrm{U}=\operatorname{diag}(\mathrm{U} 1, \mathrm{U} 2, \mathrm{U} 3)$, where $\mathrm{U} 1=\operatorname{diag}(0.8,0.9,0.9,0.7,0.8,1,1)$,

$\mathrm{U} 2=\operatorname{diag}(0.9,0.8,0.8,0.7), \mathrm{U} 3=\operatorname{diag}(1,0.9)$.

The input and output matrices is improved to $\mathbf{I}=\left[\begin{array}{ccc}\mathbf{I}_{1} & \mathbf{0} & \mathbf{0} \\ \mathbf{0} & \mathbf{I}_{2} & \mathbf{0} \\ \mathbf{0} & \mathbf{0} & \mathbf{I}_{3} \\ \mathbf{0} & \mathbf{0} & \mathbf{0}\end{array}\right], \mathbf{O}=\left[\begin{array}{ccc}\mathbf{0} & \mathbf{0} & \mathbf{0} \\ \mathbf{\mathbf { O } _ { 1 }} & \mathbf{0} & \mathbf{0} \\ \mathbf{0} & \mathbf{O}_{2} & \mathbf{0} \\ \mathbf{0} & \mathbf{0} & \mathbf{O}_{3}\end{array}\right]$.

The FPN reasoning decision in Section 1.3 takes a 0 as a known quantity to iterate out ak +1 , as actually as takes a01 as a known quantity to iterate out a02, a03 and a04 in turn. Each time the value of $\mathrm{k}$ is added, the following vector will be obtained. Therefore, the combination of $\mathrm{a} 01, \mathrm{a} 02, \mathrm{a} 03$ and $a 04$ is the final state. The result is $a 1=(a 01, a 02, a 03, a 04)$.

The following steps are used to calculate a 02 :

Step $1: \mathrm{a} 01=(0.75,0.3,0.6,0.7,0,0.8,0.6,0.6)$.

Step 2:nega01 $=(0.25,0.7,0.4,0.3,1,0.2,0.4,0.4)$.

Step 3:v01 $=\mathrm{IT} 1 \otimes($ nega01 $)=(0.25,0.7,0.4,1,0.2,0.4,0.4)$.

Step 4: $01=$ negv01 $=(0.75,0.3,0.6,0,0.8,0.6,0.6)$.

Step $5: \varepsilon 01=\rho 01 \odot \operatorname{sign}(\rho 01-\Lambda 1)=(0.75,0,0.6,0,0.8,0.6,0.6)$.

Step $6: \mathrm{a} 02=(\mathrm{O} 1 \cdot \mathrm{U} 1) \otimes \varepsilon 01=(0.6,0,0.64,0.6)$.

Similarly, a03 $=(\mathrm{O} 2 \cdot \mathrm{U} 2) \otimes \varepsilon 02=(0.54,0.512), \mathrm{a} 04=(\mathrm{O} 3 \cdot \mathrm{U} 3) \otimes \varepsilon 03=(0.54)$.

The final library state is $\mathrm{a} 1=(\mathrm{a} 01, \mathrm{a} 02, \mathrm{a} 03, \mathrm{a} 04)=(0.75,0.3,0.6,0.7,0,0.8,0.6,0.6,0.6,0,0.64$, $0.6,0.54$,

$0.512,0.54)$.

It can be concluded that the fault probability of thread feedback error is 0.54 , the fault probability of measurement without data or fluctuation is 0.512 , and the fault probability of terminal control alarm is 0.54 .Comparing the fault probability of these three items in the actual detection phenomenon, it shows that the accuracy of fault probability is improved.

\section{Summary}

In this paper, fuzzy Petri nets is used to express the fault information and the direction of fault spread of smart substation equipment, and the hierarchical modeling method is used to make the logic relation more intuitive and simple. It is convenient to locate fault information and fault source accurately. On the basis of traditional FPN reasoning, the transition threshold judgment is increased, and the accuracy of fault probability is further improved. The establishment of hierarchical modeling method simplifies the reasoning process and operation structure of the diagnostic method, and improves the feasibility and effectiveness of the algorithm. Through the calculation of the actual data of some devices in smart substation, it can be seen that the method has good applicability. 
Of course, the initial state and confidence equivalent of this paper are based on the abstract data in practical application, and the setting of the transition threshold is also subjective, which needs further study.

\section{References}

[1]. BIAN Li,BIAN Chen-yuan.Review on Intelligence Fault Diagnosis in Power Networks[J].Power System Protection and Control,2014,42(03):146-153.

[2]. Chengyan Xu,Yan Bai,Renshu Wang. The Fault Diagnosis of The Wireless Sequence Control System Based on Fuzzy Petri Nets[J].Computer Engineering \& Science,2014,36(08):1512-1518.

[3]. Dai Kong,Hongwei Li.Fuzzy Petri Nets and Its Application in Fault Diagnosis of Compressor[J].Computer Engineering and Design,2018,39(01):271-275.

[4]. Qiang Chen, Xuezhen Cheng, Jianhang Liu, et al. The Analysis Method of Power Grid Fault Based on Hierarchical Transition Weighted Fuzzy Petri Net [J].Transactions of China Electrotechnical Society, 2016, 31(15):125-135.

[5]. Jiangwei Yang, Zhengyou He. The Fault Diagnosis of Power Grid Based on Temporal Order Fuzzy Petri Nets [J].Automation of Electric Power Systems, 2011, 35(15):46-51.

[6]. Zhihua Cai.Fuzzy Petri Nets And Knowledge Representation[J].Computer Applications and Software, 1994(03):30-36.

[7]. Jianwei Yang,Zhengyou He,Tianlei Zang.Power System Fault-diagnosis Method Based on Directional Weighted Fuzzy Petri Nets[J].Proceedings of the CSEE,2010,30(34):42-49.

[8]. Hongtao Xie,Xiaoyang Tong.A Method of Synthetical Fault Diagnosis for Power System Based on Fuzzy Hierarchical Petri Net[J].Power System Technology, ,2012,36(01):246-252.

[9]. Guojun Xu,Yongsheng Liu,Liang Hua,et al.Application of Smart Substation Fault Diagnosis Based on Fuzzy Cause-effect Networks[J].Power System Protection and Control,2013,41(08):93-98.

[10]. Daoyin Zhang,Xiao-fei Zhang,Ruying Zhao.Research on Fault diagnosis Technology of Smart Substation[J].Electric Power Information and Communication Technology,2013,11(07):39-43.

[11]. Ludwik Czaja,Zbigniew Suraj.A New Class of Fuzzy Petri Nets for Knowledge Representation and Reasoning[J].Fundamenta Informaticae,2013,128(1-2).

[12]. Alireza Tavakholi Ghainani,Abdullah Asuhaimi Mohd Zin,Nur'Ain Maiza Ismail,Zheng-Guang Wu.Fuzzy Timing Petri Net for Fault Diagnosis in Power System[J].Mathematical Problems in Engineering,2012,2012.

[13]. Liangbing Feng,Masanao Obayashi,Takashi Kuremoto,Kunikazu Kobayashi.A learning Fuzzy Petri net model[J].IEEJ Transactions on Electrical and Electronic Engineering,2012,7(3). 